\title{
Indeterminate Domain Proteins Regulate Rice Defense to Sheath Blight Disease
}

\author{
Qian Sun ${ }^{1+}$, Dan Dan $\mathrm{Li}^{1 \dagger}$, Jin Chu' ${ }^{2 \dagger}$, De Peng Yuan', Shuang Lij ${ }^{3,4}$, Li Juan Zhong ${ }^{5}$, Xiao Han ${ }^{6 *}$ and \\ Yuan Hu Xuan ${ }^{1 *}$ (D)
}

\begin{abstract}
Background: Loose Plant Architecture 1 (LPA1), an indeterminate domain (IDD) protein, exhibits almost no expression in the leaves, but the overexpression of LPA1 significantly increases the resistance of rice to sheath blight disease (ShB) via the activation of PIN-FORMED 1a (PIN1a).

Results: In this study, we determined that Rhizoctonia solani infection significantly induced LPA1 expression in the leaves, and Ipal was more susceptible to $R$. solani compared with the wild-type and revertant plants. In addition, infection with $R$. solani altered the expression of IDD3, IDD5, IDD10, and IDD13, and yeast two-hybrid, split-GFP, and coimmunoprecipitation assays showed that LPA1 interacts with IDD3 and IDD13. IDD13 RNAi plants were more susceptible, while IDD13 overexpressors were less susceptible to ShB compared with the wild-type. In parallel, idd3 exhibited no significant differences, while IDD3 overexpressors were more susceptible compared to the wild-type response to ShB. Additional chromatin-immunoprecipitation and electrophoretic mobility shift assay experiments indicated that IDD13 and IDD3 bound to the PIN1a promoter, and the transient assay indicated that IDD13 and IDD3 positively and negatively regulate PIN1a expression, respectively. Moreover, IDD13, IDD3, and LPA1 form a transcription factor complex that regulates PIN1a. A genetic study showed that the LPA1 repressor lines were similar to Ipa1/IDD13 RNAi and were more susceptible than the Ipa1 and IDD13 RNAi plants in response to ShB. The overexpression of IDD13 increased resistance to ShB in the lpal background.
\end{abstract}

Conclusions: Taken together, our analyses established that IDD3, IDD13, and LPA1 form a transcription factor complex to regulate the defense of rice against ShB possibly via the regulation of PIN1a.

Keywords: Indeterminate domain protein, Sheath blight disease, Transcription activation, Defense, Rice

\section{Background}

Sheath blight disease (ShB) is one of the three major diseases that are caused by Rhizoctonia solani in rice (Oryza sativa) (Savary et al., 1995). The fungus damages rice during the whole period of the growth cycle and primarily infects the leaves, sheaths, and panicles. At the late stage of infection, the whole plant withers and lodges (Savary et al., 1995). ShB can reduce rice yield production up to $50 \%$ when the disease is severe (Savary et al. 2000). Since there is a lack of resistant cultivars against $\mathrm{ShB}$, the application of fungicides is the current

\footnotetext{
* Correspondence: xiaohan@caas.cn; xuanyuanhu115@syau.edu.cn ${ }^{+}$Qian Sun, Dan Dan Li and Jin Chu contributed equally to this work. ${ }^{6}$ College of Biological Science and Engineering, Fuzhou University, Fuzhou 350108, China

${ }^{1}$ College of Plant Protection, Shenyang Agricultural University, Shenyang 110866, China

Full list of author information is available at the end of the article
}

primary approach to control this disease (Savary et al. 2000). However, its use severely influences environmental conditions because of its effect on microbes in the environment, and the fungicides also increase the cost of cultivation. Thus, the isolation of resistant rice cultivars and the exploration of defense mechanisms against SbB have become an important issue. Previous studies have demonstrated that the overexpression of chitinase, $\beta-1,3$ glucanase, or polygalacturonase-inhibiting protein (OsPGIP1) enhances the resistance of rice to $R$. solani (Shah et al. 2009; Mao et al. 2014; Wang et al. 2015). The overexpression of an ethylene synthesis enzyme (OsACS2) promotes the resistance of rice to blast and sheath blight (Helliwell et al. 2013). The overexpression of BROADSPECTRUM RESISTANCE2 (BSR2) resulted in resistance to $R$. solani in Arabidopsis and rice (Maeda et al. 2019), and salicylic acid-dependent immunity contributes to resistance against $R$. solani in rice and Brachypodium 
distachyon (Kouzai et al. 2018). In addition, our recent studies identified that a mutation in Sugar Will be Eventually be Exported Transporter 11 (SWEET11) significantly promoted the defense of rice to ShB (Gao et al. 2018); Related to ABI3/VP1-Like 1 (RAVL1) modulates rice defense against $\mathrm{ShB}$ via the activation of brassinosteroids and ethylene signaling genes (Yuan et al. 2018), and the overexpression of LPA1 (IDD14) promoted the defense of rice against ShB via the activation of PIN1a (Sun et al. 2019).

The indeterminate domain (IDD) consists of two $\mathrm{C}_{2} \mathrm{H}_{2}$ and two $\mathrm{C}_{2} \mathrm{HC}$ zinc finger motifs, and the IDD genes play diverse biological functions in plants. ID1 has been reported to control the flowering time in maize and rice (Colasanti et al. 1998; Park et al. 2008). Magpie (MAG)/ AtIDD3 and jackdaw (JKD)/AtIDD10 regulate the fate of root cells (Welch et al. 2007). Enhydrous (ENY)/AtIDD1 regulates seed maturation (Feurtado et al. 2011). AtIDD8 modulates plant development (Seo et al. 2011). AtIDD14, AtIDD15, and AtIDD16 cooperatively regulate lateral organ morphogenesis and gravitropism by promoting auxin biosynthesis and transport in Arabidopsis (Cui et al. 2013). Loose plant architecture1 (LPA1)/IDD14 regulates shoot gravitropism and lamina joint angle (Wu et al. 2013; Liu et al. 2016). The regulator of CBF1 (ROC1)/IDD3 activates $\mathrm{DREB} 1 \mathrm{~B} / \mathrm{CBF} 1$ to regulate chilling tolerance in rice (Dou et al. 2016). IDD2 regulates secondary cell wall formation in rice (Huang et al. 2018). In addition, the AtIDD4 repressor constitutively induces immunity in Arabidopsis (Volz et al. 2019). The binding motifs of the transcription factor IDD have been identified in maize (ID1,

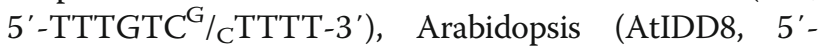
TTTTGTCC-3'), and rice (IDD10, $5^{\prime}-$ TTTGTC $^{\mathrm{C}} / \mathrm{G}$ ) (Kozaki et al. 2004; Seo et al. 2011; Xuan et al. 2013). However, the function of IDD in plant defense, as well as the IDD target genes, remains largely unknown.

Auxin is one of the key phytohormones, and its polar transport is regulated by auxin influx AUX1/LAX and efflux protein PINs (Adamowski and Friml, 2015; Zazimalova et al. 2010). Auxin plays key roles in plant growth and development, as well as in controlling plant defense (Robert-Seilaniantz et al. 2011; Naseem et al. 2012; Chen et al. 2007). More studies identified that auxin signaling regulates rice defense against the bacterial pathogen Xanthomonas oryzae (Fu et al. 2011) and the fungal pathogen Magnaporthe oryzae (Fu et al. 2011). Recently, we identified that exogenously treated auxin increases the resistance of rice to $R$. solani AG1-IA and revealed that LPA1 overexpression activates PIN1a to promote defense against $R$. solani in rice (Sun et al. 2019). However, whether other IDDs regulate the resistance of rice to $\mathrm{ShB}$ remains to be elucidated. In this study, we performed molecular, biochemical, and genetic studies to explore the function of IDD in rice defense. The results showed that IDD3 and IDD13 interact with LPA1 to regulate PIN1a expression and act to modulate the resistance of rice to ShB. Taken together, our analyses provide information on the role of the IDDs in the regulation of rice defense, as well as the regulatory mechanism for $\mathrm{ShB}$ in rice.

\section{Results \\ LPA1 Is Induced by Rhizoctonia solani, and Ipa1 Is more Susceptible to Sheath Blight Disease}

Previously we demonstrated that the overexpression of LPA1 significantly promotes the resistance of rice to $\mathrm{ShB}$ via the activation of PIN1a (Sun et al. 2019). However, previous research indicated that LPA1 was expressed at very low levels in the leaves and sheath (Wu et al. 2013). Therefore, we analyzed the $R$. solani infection-dependent LPA1 expression in more detail. Interestingly, infection with $R$. solani significantly induced $L P A 1$ expression after $72 \mathrm{~h}$ (Fig. 1a). Examination of the response of lpa1, LPA1 revertant (Rev.), and wild-type (WT) plants (Liu et al. 2016) showed that lpa1 was more susceptible to $R$. solani AG1-IA than the WT and revertant (Rev.) plants (Fig. 1b). The percentage of the leaf area covered with lesions was $41 \%$ in the WT, 56\% in lpa1, and 39\% in Rev. (Fig. 1c). Since LPA1 activates PIN1a transcription, the PIN1a expression level was examined in the wild-type, lpal, and Rev. plants before and after inoculation with $R$. solani AG1-IA. The qRT-PCR results showed that PIN1a was induced by $R$. solani AG1-IA inoculation, and the level was lower in lpa1 than in the wild-type and Rev. plant leaves $72 \mathrm{~h}$ post- $R$. solani inoculation, but there were no significant differences without the inoculation (Fig. 1d).

\section{IDD13 and IDD3 Interact with LPA1}

Our transcriptome study discerned that several IDD genes, including $I D D 3$, IDD5, $I D D 10$, and $I D D 13$, were differentially expressed upon $R$. solani infection (unpublished data). To verify the transcriptome data, qRT-PCR was performed to examine the expression of the $I D D$ gene. The results showed that $I D D 5$ was suppressed, while $I D D 3, I D D 10$, and $I D D 13$ were induced by $R$. solani (Fig. 2a). To test whether IDD3, IDD10, or IDD13 interact with LPA1, yeast two-hybrid, split-GFP, and co-immunoprecipitation (co-IP) assays were performed. A yeast-two hybrid analysis indicated that LPA1 interacts with IDD3 and IDD13 but not with IDD10 (Fig. 2b). An additional split-GFP assay showed that LPA1 interacts with IDD3 or IDD13 at the nucleus in $N$. benthamiana leaves, but the negative control (LPA1-nYFP+cCFP) did not exhibit a visible signal (Fig. 2c). For the co-IP assay, LPA1-GFP was co-expressed with IDD3-Myc, IDD13-Myc or IDD10-Myc in N. benthamiana leaves, and the total proteins were immunoprecipitated using an anti-GFP antibody. The immunoprecipitated proteins were analyzed using an anti-Myc antibody. The results indicated that LPA1 interacts with IDD3 and IDD13 but not IDD10 in plants, and the interaction affinity in LPA1-IDD13 was higher than that in LPA1-IDD3 (Fig. 2d). 

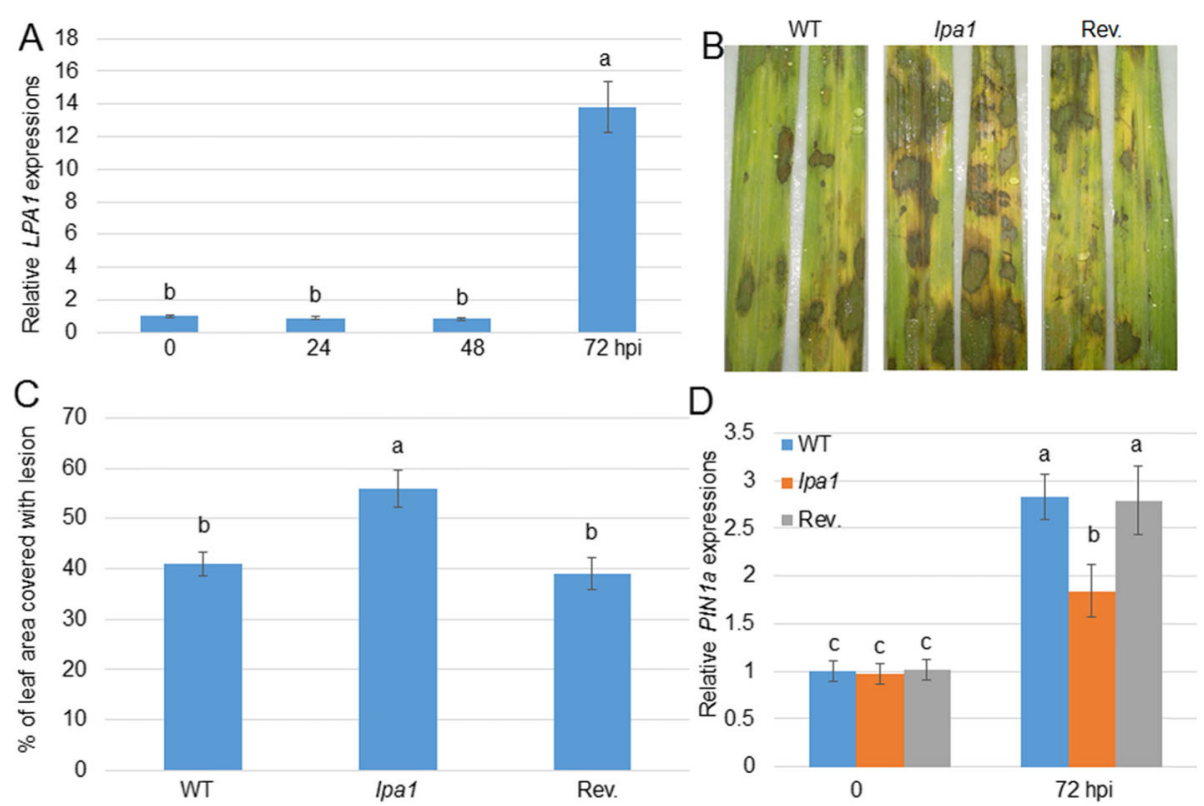

Fig. 1 Rhizoctonia solani-mediated LPA1 expression patterns and Ipa1 mutant response to sheath blight disease. a $L P A 1$ expression level in the leaves at $0,24,48$, and $72 \mathrm{~h}$ post-inoculation (hpi) of $R$. solani AG1-IA. The error bars are the mean $\pm \mathrm{SE}(n=3)$. $\mathbf{b}$ Response of Ipa1 and revertant (Rev.) to $R$. solani AG1-IA compared with the wild-type (WT). c Percentage of the leaf area covered with lesions in Ipal and revertant (Rev.) compared with the WT. Data represent means \pm SE $(n>10)$. $\mathbf{d} R$. solani-mediated expression of PIN1 $a$ in WT, Ipal, and Rev. leaves before and after 72 hpi of $R$. solani. Different letters indicate significant differences at $P<0.05$

\section{IDD3 Negatively and IDD13 Positively Regulated Resistance to Sheath Blight Disease}

To analyze the function of IDD3 and IDD13 in response to $\mathrm{ShB}$, idd3 mutants (idd3-1 and idd3-2), IDD3 overexpressors (OX), IDD13 RNAi, and IDD13 OX plants were tested. Before examining their response to $\mathrm{ShB}$, the levels of expression of IDD3 and IDD13 were analyzed. The qRT-PCR results showed that the IDD3 transcript was not detected in two idd3 knock-out mutants (idd31 and $i d d 3-2)$, while IDD3 was highly expressed in the IDD3 OX plants (\#2, \#3, \#4, and \#6) compared with the wild-type control (Fig. 3a). In addition, IDD13 was significantly suppressed in the IDD13 RNAi lines (\#1, \#2, $\# 4$, and \#5), while it was obviously highly expressed in the IDD13 OX plants (\#2, \#3, \#5, and \#7) compared with the wild-type control (Fig. $3 \mathrm{~b}$ ). An additional $R$. solani infection test showed that the idd3 mutants were similar to the wild-type control and displayed a susceptible response to $R$. solani AG1-IA, but IDD3 OX exhibited more susceptible symptoms than those in the wild-type plants. The percentage of the leaf area covered with lesions was $41 \%$ in WT, $42 \%$ in idd $3-1,40.5 \%$ in idd3-2, $54.5 \%$ in IDD3 OX \#2, and 56\% in IDD3 OX \#4 plants (Fig. 3c and d). In addition, the $R$. solani infection results indicated that the IDD13 RNAi plants were more susceptible, while the IDD13 OX plants were less susceptible to ShB than the wild-type control. The percentage of leaf area covered with lesions was $39 \%$ in the WT, $48 \%$ in IDD13 RNAi \#1, 49\% in IDD13 RNAi \#4,31\% in IDD13 OX \#2, and 30\% in IDD13 OX \#5 plants (Fig. 3e and f).

\section{IDD3 and IDD13 Directly Regulate PIN1a Transcription}

LPA1 promotes the resistance of rice to $\mathrm{ShB}$ via the activation of PIN1a (Sun et al., 2019), and IDD3 and IDD13 interact with LPA1 to regulate the resistance to $\mathrm{ShB}$. Therefore, we tested the potential of IDD3 and IDD13 to bind to the PIN1a promoter in more detail using a chromatin immunoprecipitation (ChIP) assay. Before performing the ChIP assay, the IDD3-GFP and IDD13-GFP localization in the transgenic plants was evaluated. The GFP signal was detected in the nucleus of IDD3-GFP and IDD13-GFP transgenic lateral roots (Fig. 4a). In the PIN1a promoter region, a single IDD-binding motif was identified (Fig. 4b). To examine whether IDD3 and IDD13 bind to the IDD-binding motif, a ChIP assay was performed using 35S:IDD3:GFP or 35S:IDD13:GFP transgenic plant calli and an anti-GFP antibody. The samples without the application of the GFP antibody (-Ab) were used as the control for the GFP antibody $(+\mathrm{Ab})$ to immunoprecipitate the DNA. The ChIP-PCR results showed that IDD3 and IDD13 bound to the P2 but not to the P1 (Fig. 4c). An electrophoretic mobility shift assay (EMSA) was performed to verify that IDD3 and IDD13 bound the P2 fragment. The results indicated that IDD3 and IDD13 bound to P2, but the complex failed to bind to the mutated putative IDD-binding motif (TTTGTCG mutated to AAAAAAA) mP2 (Fig. 4d). To verify 

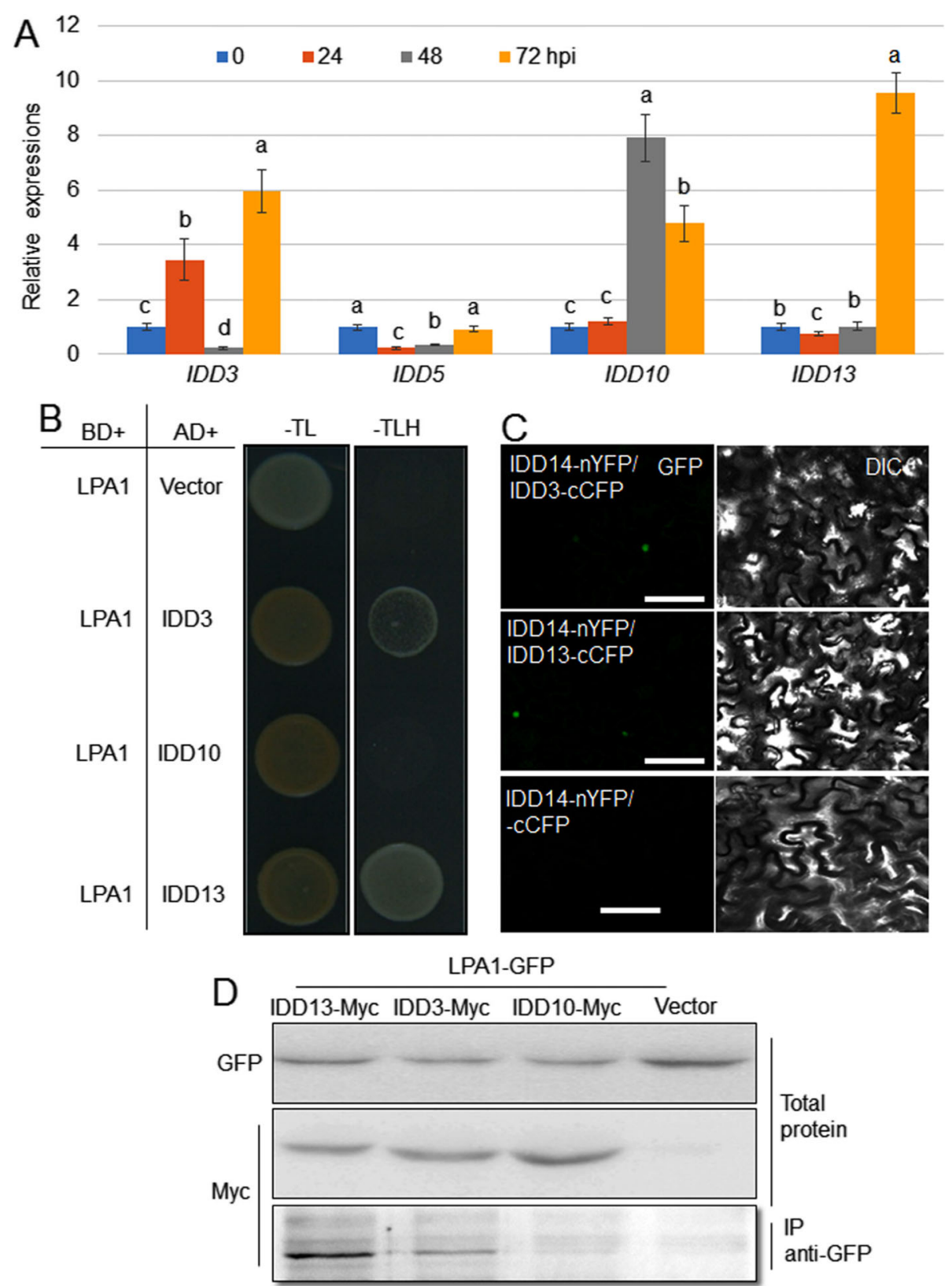

Fig. 2 Interaction between LPA1 and IDD3 or IDD13. a IDD3, IDD5, IDD10, and IDD13 expression levels in the leaves at 0, 24, 48, and 72 h postinoculation (hpi) of Rhizoctonia solani AG1-IA. The error bars are the mean \pm SE $(n=3)$. The statistical analysis was performed for each gene, and different letters indicate significant differences at $P<0.05$. $\mathbf{b}$ A yeast two-hybrid assay was performed to analyze the interaction between LPA1 and IDD3, IDD10, or IDD13. BD: GAL4-DNA binding domain; AD: activation domain; -T: without tryptophan; -L: without leucine; $-\mathrm{H}$ : without histidine. $\mathbf{c}$ Reconstitution of GFP fluorescence from LPA1-nYFP + IDD3-cCFP, LPA1-nYFP + IDD13-cCFP, and LPA1-nYFP + cCFP. Bars $=10 \mu \mathrm{m}$. DIC: differential interference contrast. d A co-IP assay was performed to analyze the interaction between LPA1 and IDD3, IDD13, or IDD10 in tobacco leaves. IDD3-Myc, IDD13-Myc, IDD10-Myc + LPA1-GFP, or empty vector + LPA1-GFP were transformed into tobacco leaves using Agrobacteriummediated transformation. Green fluorescent protein (GFP) antibody-immunoprecipitated proteins were analyzed using western blot analysis with the Myc antibody. IDD3-Myc, IDD13-Myc, IDD10-Myc, and LPA1-GFP levels were analyzed by western blot using Myc and GFP antibodies, respectively

the IDD3 and IDD13 activation of PIN1a via binding to the P2 region in the promoter, transient expression assays were conducted using the protoplast system. Protoplast cells were co-transformed with the 35S:IDD3 or 35S:IDD13 plasmid and the construct expressing the ß-glucuronidase gene (GUS) under the control of pPIN1a or mpPIN1a. In the mutated promoter (mpPIN1a), the IDD-binding motif sequences TTTGTCG were replaced with AAAAAAA. Protoplast cells 


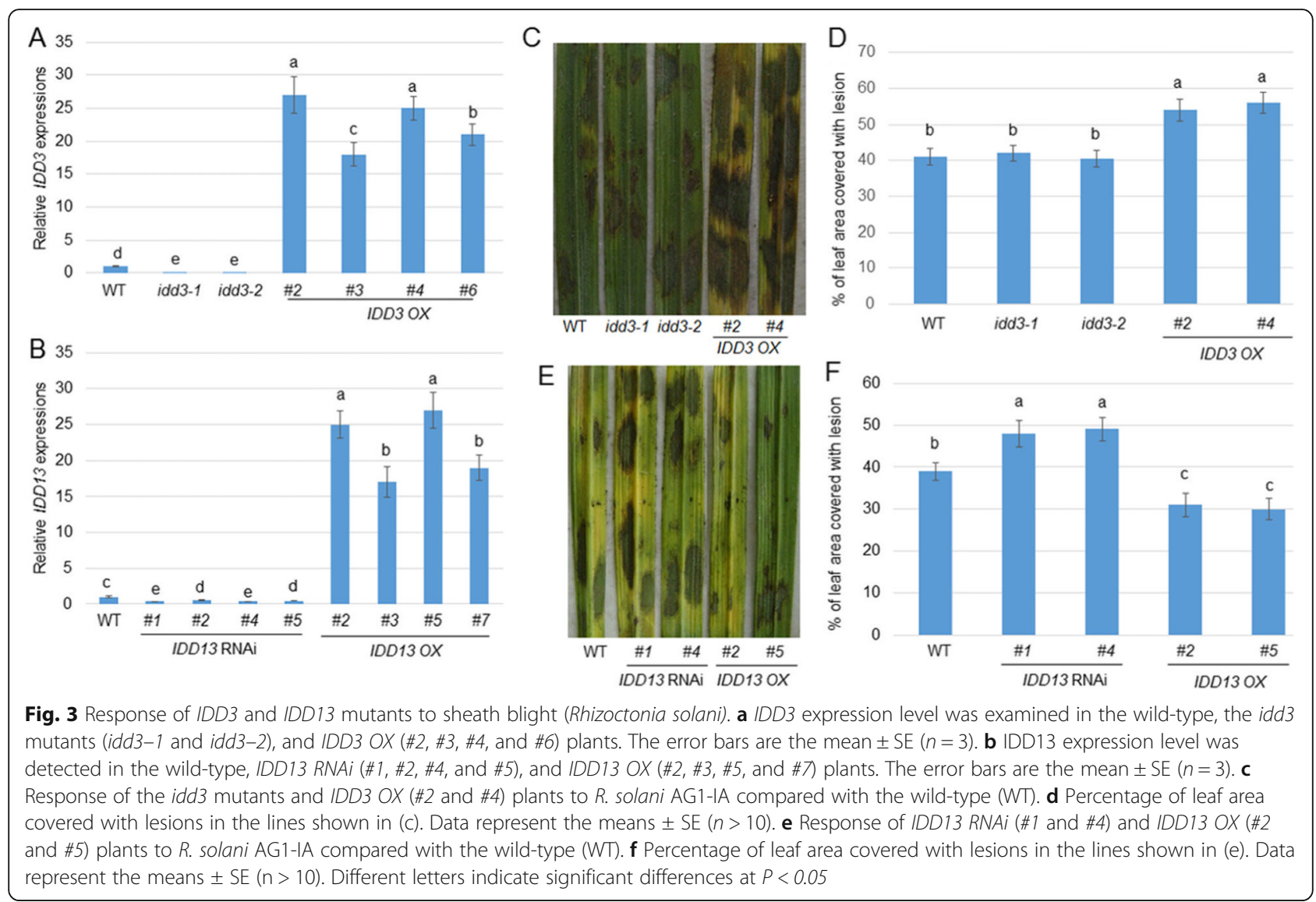

expressing IDD13 had approximately twice the levels of activated pPIN1a. However, IDD13 was unable to activate mpPIN1a. In parallel, IDD3 suppressed pPIN1a by approximately one third but did not affect mpPIN1a (Fig. 4e).

In addition, the PIN1a expression level was examined in the idd3 mutants and IDD3 OX, as well as in the IDD13 RNA $i$ and IDD13 OX plants. The qRT-PCR results showed that the PIN1a level was obviously lower in IDD3 OX than in the wild-type and idd3 mutants, but there were no significant differences between the wildtype and idd3 mutants (Additional file 1: Figure S1a). Moreover, the PIN1a level was slightly lower in the IDD13 RNAi plants, while it was significantly higher in the IDD13 OX plants than in the wild-type control (Additional file 1: Figure S1b).

\section{IDD3 Inhibits the LPA1-Mediated Activation of PIN1a Expression}

IDD3 and IDD13 interact with LPA1, and IDD13 and LPA1 directly activate PIN1a transcription, while IDD3 suppresses it. Therefore, the effects of IDD3 on IDD13 and LPA1 regulation on PIN1a expression were examined. To verify the effect, 35S:LPA1 was co-transformed with 35S:IDD13 or 35S: IDD3 and a vector expressing GUS under the control of pPIN1a. The results indicated that IDD13 and LPA1 activated pPIN1a, while IDD3 suppressed pPIN1a. In addition, co-expressing IDD13 and LPA1 increased the activation of pPIN1a compared to expressing the single IDD protein. However, the expression of IDD3 inhibited the LPA1 activation of pPIN1a (Fig. 5a). In addition, the possibility that IDD3, IDD13, and LPA1 form a transcriptional complex was tested. IDD3-HA and IDD13-Myc were expressed in $N$. benthamiana leaves and immunoprecipitated using an anti-HA antibody, but the western blot results indicated that IDD3 did not interact with IDD13 (Fig. 5b). Additional IDD3-HA, IDD13-Myc, and LPA1-GFP proteins were expressed in $N$. benthamiana leaves, and the total protein was immunoprecipitated with an anti-HA antibody. The CoIP results showed that IDD3, IDD13, and LPA1 form a transcriptional complex in plants (Fig. 5b).

IDD13 Additively Functions with LPA1 in the Regulation of Resistance to Sheath Blight Disease

The IDD13 RNAi and lpal mutants were more susceptible to $\mathrm{ShB}$, while the idd3 mutants exhibited no significant differences compared to the wild-type control, suggesting that IDD13 and LPA1 but not IDD3 might play a major role in the resistance of rice to $\mathrm{ShB}$. To analyze whether $I D D 13$ and $L P A 1$ are functionally additive in the regulation of the resistance of rice to $\mathrm{ShB}$, two genetic combinations were generated, 

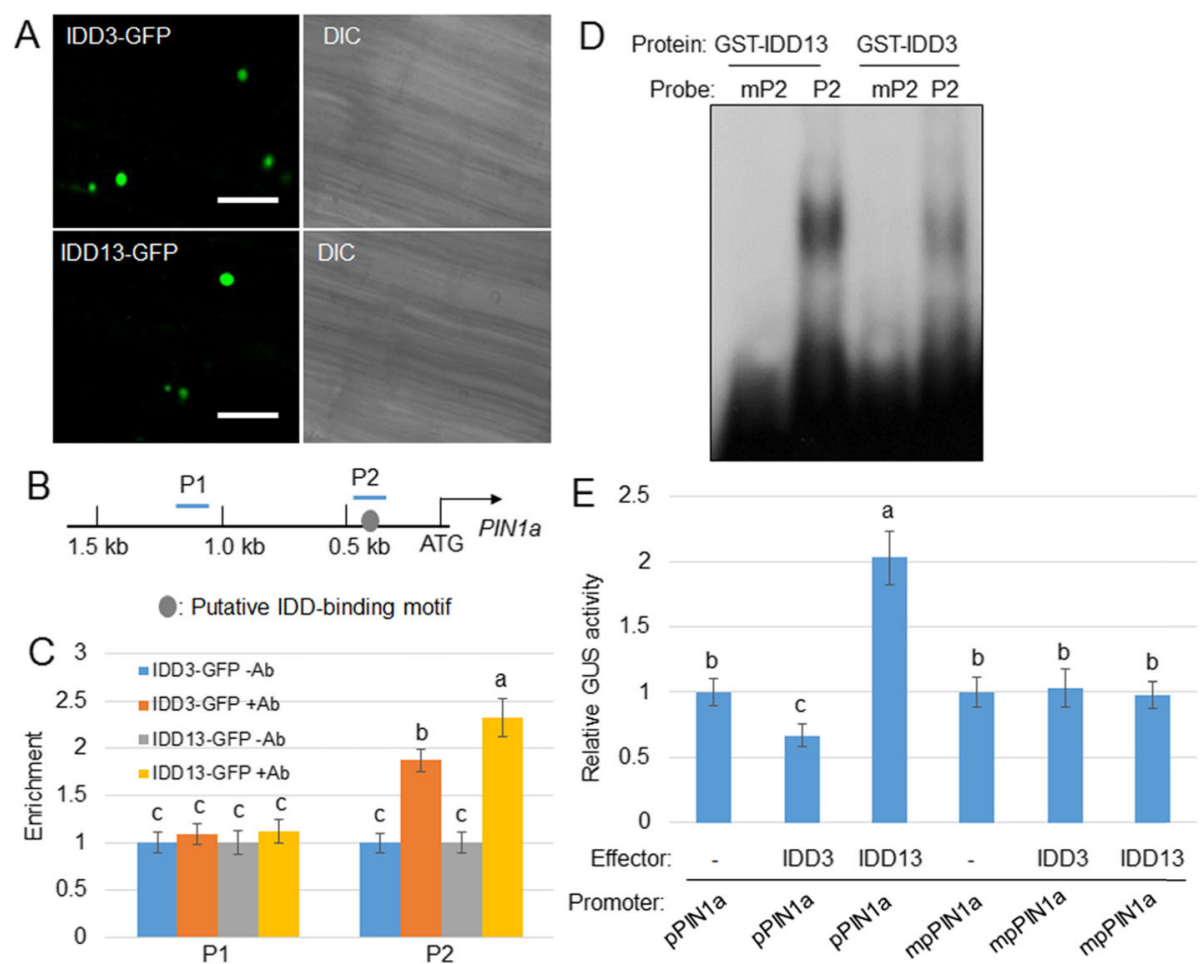

Fig. 4 IDD3 and IDD13 bind and activate the PIN1a promoter. a IDD3-GFP and IDD13-GFP were detected in the lateral roots. GFP signal and bright field are shown in the left and right, respectively. Bars $=20 \mu \mathrm{m}$. $\mathbf{b}$ Schematic diagram indicating the location of the putative IDD-binding motif (gray circle) within $1.5 \mathrm{~kb}$ of the PIN1 a promoter and the probes (P) used for chromatin immunoprecipitation (ChIP) assays. c Relative ratios of immunoprecipitated DNA to input DNA were determined by qPCR. Input DNA was used to normalize the data. -Ab or + Ab: green fluorescent protein (GFP) antibody. Error bars represent the mean \pm SE $(n=3)$. $\mathbf{d}$ An electrophoretic mobility-shift assay (EMSA) was conducted to evaluate GST-IDD3 and GST-IDD13 affinities to P2 and mutated probe MP2. e A transient expression assay was conducted by co-transfection with p35S:IDD3 or p35S:IDD13 and each of the vectors expressing GUS under the control of native (pPIN1a) and IDD-binding motif-mutated (mpPIN1a) PIN1a promoters in protoplast cells. The luciferase gene driven by the 355 promoter was used as an internal control to normalize GUS expression. Error bars represent the mean \pm SE $(n=6)$. Different letters indicate significant differences at $P<0.05$
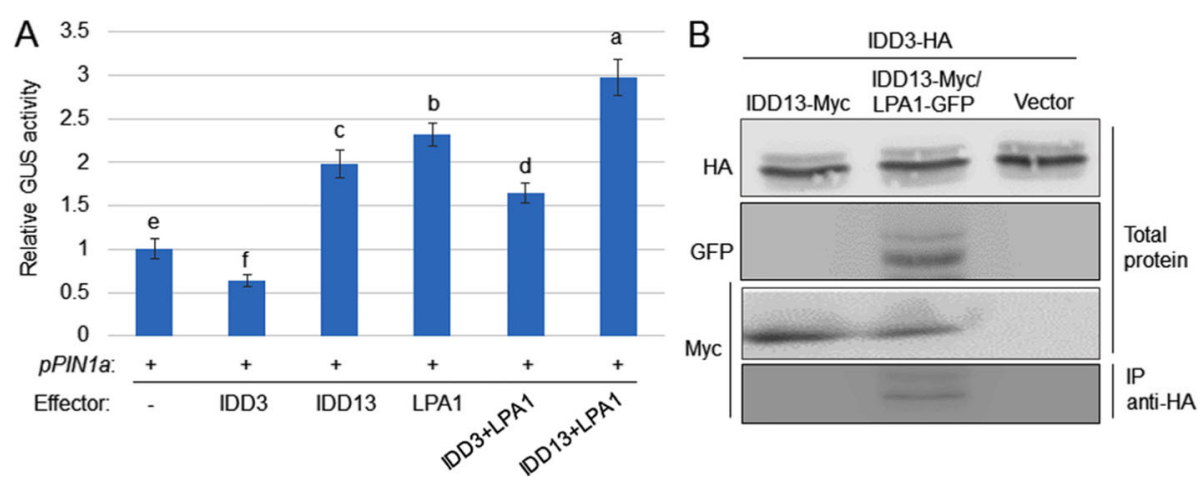

Fig. 5 IDD3, IDD13, and LPA1 form a complex to regulate PIN1a transcription. a A transient expression assay was conducted by co-transfection with p35S:IDD3, p35S:IDD13, p35S:LPA1, p35S:IDD3 + p35S:LPA1, p35S:IDD13 + p355:LPA1 and the vector expressing the GUS under the control of native (pPIN1a) PIN1a promoters in protoplast cells. The luciferase gene driven by the $35 \mathrm{~S}$ promoter was used as an internal control to normalize the GUS expression. Error bars represent the mean $\pm \mathrm{SE}(n=6)$. Different letters indicate significant differences at $P<0.05$. $\mathbf{b}$ IDD3-HA + IDD13-Myc, IDD3-HA + IDD13-Myc + LPA1-GFP, or IDD3-HA + empty vector were transformed into tobacco leaves using Agrobacterium-mediated transformation. HA antibody-immunoprecipitated proteins were analyzed using western blot analysis with the Myc antibody. IDD3-HA, IDD13Myc, and LPA1-GFP levels were analyzed by a western blot using HA, Myc, and GFP antibodies, respectively 
including IDD13 RNAi/lpa1 and lpa1/IDD13 OX. In addition, LPA1 repressor lines were examined (Wu et al. 2013, Liu et al. 2016). An $R$. solani infection test showed that IDD13 RNAi/lpa1 was more susceptible than the IDD13 RNAi, lpa1, and wild-type plants segregated from the same sibling, and IDD13 RNAi/lpa1 exhibited similar susceptible symptoms to the $L P A 1$ repressor (Fig. 6a). The percentage of the leaf area covered with lesions was $41 \%$ in the WT, $51 \%$ in IDD13 RNAi, 54\% in lpa1, 63\% in IDD13 RNAi/lpa1, and 61.5\% in the $L P A 1$ repressor plants (Fig. $6 \mathrm{~b}$ ). In addition, $R$. solani infection results indicated that the lpa1/IDD13 OX plants were less susceptible to $\mathrm{ShB}$ than the lpa1 mutant and wild-type segregated from the same sibling, but they were more susceptible to ShB compared to the IDD13 OX plants segregated from the same sibling (Fig. 6c). The percentage of leaf area covered with lesions was $40.5 \%$ in WT, 53\% in lpa1, 32\% in IDD13 OX, and 37.5\% in lpa1/IDD13 OX plants (Fig. 6d).

In addition, the level of expression of PIN1a was examined in the IDD13 RNAi/lpa1, lpa1/IDD13 OX, and LPA1 repressor lines. The qRT-PCR results showed that the PIN1a level was much lower in IDD13 RNAi/lpa1 than in the wild-type,
IDD13 RNAi, and lpa1 and was similar between IDD13 RNAi/lpa1 and the LPA1 repressor after $R$. solani inoculation (Additional file 2: Figure S2a). In parallel, the PIN1a level was higher in lpa1/IDD13 OX than in lpa1 and higher than in the wild-type plants. The PIN1a level was noticeably higher in IDD13 OX than in the wild-type and lpa1/IDD13 $\mathrm{OX}$ after R. solani inoculation (Additional file 2: Figure $\mathrm{S} 2 \mathrm{~b}$ ).

\section{IDD13 Overexpresssion Maintained Yield Production in}

Rice

Since the IDD13 OX plants demonstrated increased resistance to $\mathrm{ShB}$, yield factors were investigated further. The results demonstrated that IDD13 OX plants developed a similar tiller number, thousand-grain weight, and number of spikelets per panicle relative to the WT, but the overexpression of IDD13 slightly decreased the tiller angle compared with the wild-type (WT) plants (Fig. 7). $L P A 1$ overexpression increased the content of 3-indole acetic acid (IAA), a natural form of auxin, and exogenous IAA treatment promoted the resistance of rice to ShB (Sun et al. 2019). Therefore, the endogenous IAA
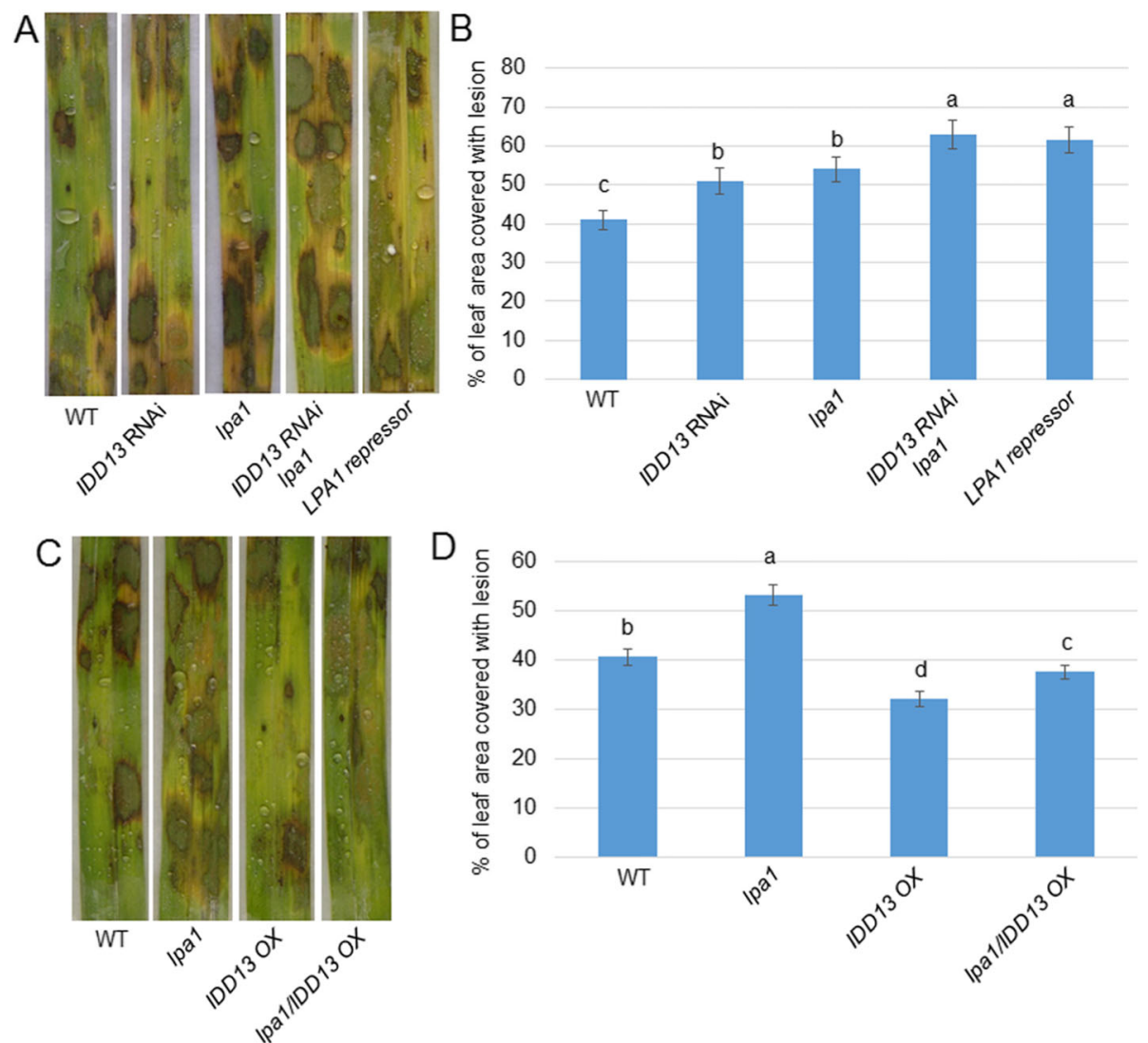

Fig. 6 IDDI3 and LPA1 genetic combinations in response to sheath blight (Rhizoctonia solani). a Response of IDD13 RNAi, Ipa1, IDD13 RNAi/Ipa, LPA1 repressor plants to $R$. solani AG1-IA compared with the wild-type (WT). $\mathbf{b}$ Percentage of the leaf area covered with lesions in the lines shown in (a). Data represent the means \pm SE $(n>10)$. c Response of the Ipa1, IDD13 OX, and Ipa1/IDD13 OX plants to R. solani AG1-IA compared with the wild-type (WT). $\mathbf{d}$ Percentage of the leaf area covered with lesions in the lines shown in (c). Data represent the means \pm SE $(n>10)$. Different letters indicate significant differences at $P<0.05$ 

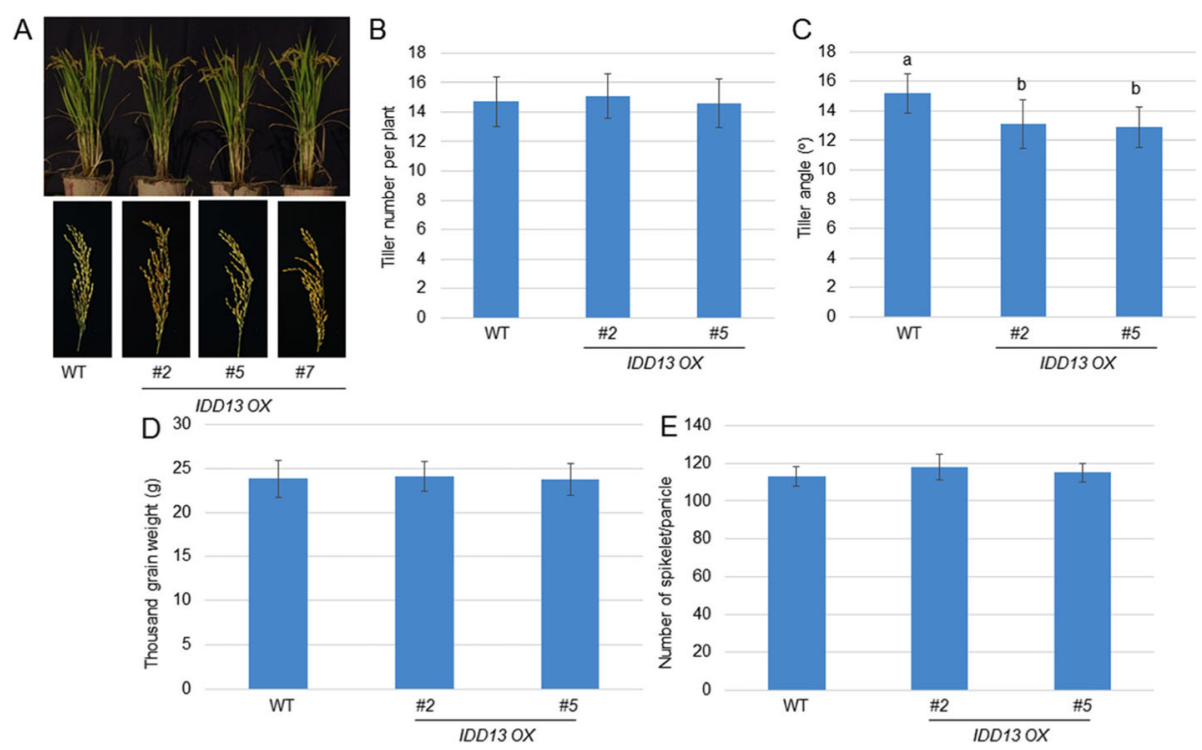

Fig. 7 The tiller number, thousand-grain weight and number of spikelet per panicle in the wild-type and IDDI3 OX plants. a The three-and-a-halfmonth-old wild-type and IDD13 OX plants (\#2, \#5, and \#7), as well as their panicles, are shown. Tiller number $\mathbf{b}$, tiller angles $\mathbf{c}$, thousand-grain weight $\mathbf{d}$, and the number of grain weight per panicle e from the wild-type and IDD13 OX plants (\#2 and \#5) were calculated. Data indicate the average $\pm \mathrm{SD}(n>15)$. Different letters indicate significant differences at $P<0.05$

levels in the WT, IDD13 OX2, and IDD13 OX5 plants were measured. The data demonstrated that IDD13 overexpressors contain higher levels of IAA than that of the WT plant leaves (Additional file 3: Figure S3).

\section{Discussion}

Sheath blight disease caused by $R$. solani is a major rice disease, which severely reduces grain yield. However, the host resistance mechanisms remain unknown. Previously, we identified that exogenous auxin treatment promoted resistance to $\mathrm{ShB}$, and the overexpression of $L P A 1 / I D D 14$ promoted rice defense to $\mathrm{ShB}$ via the activation of the auxin poplar transporter PIN1a in rice (Sun et al. 2019). PIN1a RNAi and the PIN1a overexpressors were more and less susceptible to ShB, respectively (Sun et al. 2019), suggesting that LPA1 increases the local auxin content or the activation of auxin signaling by controlling PIN1a and enhancing the resistance of the rice to ShB. However, whether IDD proteins other than LPA1 regulate the resistance of rice remains unclear.

\section{IDDs Were Induced by $R$. solani, and LPA1 Interacts with IDD3 and IDD13}

The transcriptome analysis and additional qPCR verification showed that IDD3, IDD10, IDD13, and LPA1 were upregulated, while $I D D 5$ was down-regulated by $R$. solani in rice. In normal conditions, $L P A 1$ is barely expressed in the leaves and sheath of rice (Wu et al. 2013), but $R$. solani infection significantly induced the level of expression of LPA1 in the leaves. Additional genetic study showed that the lpal and IDD13 RNAi mutant were more susceptible, but the idd3 mutants exhibited a similar response to ShB compared with the wild-type. In addition, the overexpression of $I D D 13$ produced results similar to those of $L P A 1$, whereas the overexpression of $I D D 3$ inhibited the resistance of rice to $\mathrm{ShB}$, indicating that rice defense against $\mathrm{ShB}$ requires $L P A 1$ and $I D D 13$, and IDD3 negatively regulates the defense of rice to ShB. Since AtIDD15 functions in concert with AtIDD14 and AtIDD16 to directly activate auxin biosynthesis and transportrelated genes in Arabidopsis (Cui et al. 2013), this suggests that IDD proteins are functionally additive in the regulation of auxin biosynthesis. Further biochemical and molecular assays identified that LPA1 interacts with IDD3 and IDD13, and the interaction affinity of LPA1 was higher with IDD13 than with IDD3. However, IDD3 did not directly interact with IDD13, while LPA1, IDD3, and IDD13 form a transcriptional complex, suggesting that these three IDDs may form a transcriptional complex to regulate the resistance of rice to ShB.

\section{IDD13 Positively and IDD3 Negatively Regulate PIN1a}

LPA1 and IDD10 were reported to localize to the nucleus and function as transcription factors (Wu et al. 2013; Xuan et al. 2013), and IDD3-GFP and IDD13-GFP were localized to the nucleus in the transgenic rice roots. Previously, we identified that PIN1a is a direct target of LPA1, which positively regulates the resistance of rice to ShB (Sun et al. 2019). Since LPA1 interacts with IDD3 and IDD13, the roles of IDD3 and IDD13 in the regulation of PIN1a transcription were analyzed. Additional ChIP and EMAS assays showed that IDD13 and IDD3 directly bound to the putative IDD-binding motif in the PIN1a promoter region. In addition, a transient assay 
revealed that IDD13 acted similarly to LPA1 to directly activate PIN1a, but IDD3 directly bound to the PIN1a promoter and functioned as a transcriptional repressor. The transient assay results showed that the expression of IDD13 and LPA1 activated the level of transcription of PIN1a, while the expression of IDD3 suppressed the level of expression of PIN1a, suggesting that IDD13 and LPA1 function as transcriptional activators, while IDD3 functions as a transcriptional repressor to PIN1a.

The overexpression of $L P A 1$ reduced the tiller angle and increased the contents of IAA in the leaves via the activation of PIN1a (Sun et al. 2019). IDD13 activates PIN1a, and the additional investigation of yield factors identified that the overexpression of IDD13 maintained yield production, while reducing the tiller angle compared with the wild-type plants. In addition, the IDD13 OX plants accumulated higher contents of IAA than were found in the wild-type plant leaves, suggesting that IDD13 OX acts similarly to the LPA1 OX plants to increase the resistance of rice to ShB by activating PIN1a without affecting rice production. However, the tiller angle of the LPA1 OX plants is smaller than that in the IDD13 OX plants, implying a dominant regulation of LPA1 compared with IDD13 in the transcriptional activation of PIN1a.

\section{IDD13 and LPA1 Are Functionally Additive in the Regulation of the Resistance of Rice to Sheath Blight Disease}

IDD3, IDD13, and LPA1 physically interact with and differentially regulate PIN1a. In addition, LPA1 positively regulates the resistance of rice to $\mathrm{ShB}$. Next, a genetic study was performed to analyze the functions of IDD3 and IDD13 in the control of the resistance of rice to ShB. An $R$. solani infection assay indicated that the two idd3 mutants exhibited a similar susceptible response to ShB compared with the wild-type control. In addition, the level of expression of PIN1a was not changed in the idd3 mutants, which was similar to its expression in the wild-type plants. The overexpression of IDD3 significantly suppressed the PIN1a level compared with the wild-type plants, and IDD3 OX exhibited more susceptible symptoms to $R$. solani infection than the wild-type plants. The IDD13 RNAi plants were more susceptible to $\mathrm{ShB}$, while the IDD13 OX plants were less susceptible compared with the wild-type plants. In addition, the PIN1a expression level was lower in the IDD13 RNAi and higher in the IDD13 OX plants than in the wild-type plants, suggesting that IDD13 might regulate the resistance to ShB via the activation of PIN1a. The IDD3 mutation did not change the resistance of the rice to $\mathrm{ShB}$, as well as the PIN1a expression, implying that IDD3 might not be a major regulator of PIN1a transcription.

IDD13 and LPA1 interact and activate PIN1a transcription. In addition, the IDD3 RNA $i$ and lpa1 mutants were more susceptible to $\mathrm{ShB}$, while the IDD13 $O X$ and $L P A 1 O X$ plants were less susceptible compared with the wild-type control. Further genetic and pathology experiments indicated that an IDD13 RNAi/lpa1 double mutant was more susceptible to ShB compared with IDD13 $R N A i$ and lpa1. In addition, the response of the LPA1 repressor plants to $\mathrm{ShB}$ was compared with that of IDD13 RNAi/lpa1. The results showed that expressing the $L P A 1$ repressor to inhibit the transcription complex, including LPA1, produced a similar defect in response to ShB when compared with IDD13 RNAi/lpa1, and showed more susceptible symptom than in lpa1 and IDD13 RNAi, suggesting that IDD13 and LPA1 might be functionally additive. In parallel, the lpa1/IDD13 OX double mutants were more susceptible to ShB compared with the IDD13 OX plants, but they were less susceptible to ShB compared to lpa1 and the wild-type control, suggesting that IDD13 OX can partially rescue the defect from the LPA1 mutation in response to ShB. Additional expression level analyses indicated that the level of PIN 1a was much lower in IDD13 RNAi/lpa1 or the LPA1 repressor than in IDD13 RNAi and lpa1, while it was higher in lpa1/IDD13 OX than in lpa1. These results suggest that IDD13 and LPA1 might be functionally additive in the regulation of the resistance of rice to ShB via the activation of PIN1a expression in rice.

Overall, this study identified a new IDD transcriptional complex and identified its function in the regulation of $\mathrm{ShB}$ via the regulation of PIN1a transcription. These results will broaden our understanding of the regulatory mechanism by which the IDDs regulate auxin transport and the resistance of rice to $\mathrm{ShB}$.

\section{Methods}

\section{Plant Growth and $R$. solani AG1-IA Inoculation}

Wild-type (WT) control line (Oryza sativa Japonica, cultivar Dongjin), lpa1, LPA1 revertant (Rev.), IDD13 RNAi, IDD13-GFP overexpressor (IDD13 OX), idd3-1 (PFG_ 3A-09378), idd3-2 (PFG_3A-14,411), IDD3-GFP overexpressor (IDD3 OX), lpa1/IDD13 RNAi, lpa1/IDD13 OX, and $L P A 1$ repressor plants were used. The plants were grown in a greenhouse at Shenyang Agricultural University, China, with a temperature of $23{ }^{\circ} \mathrm{C}-30{ }^{\circ} \mathrm{C}$. Onemonth-old rice plants were inoculated with $R$. solani AG1-IA (Prasad and Eizenga, 2008). In brief, a 10-cmlong piece was cut from the second youngest leaf of the main tiller and placed on moistened filter paper in a Petri dish (diameter, $36 \mathrm{~cm}$; height, $2.5 \mathrm{~cm}$ ). Each replicate comprised six leaves, and four replicates per line were used in a completely randomized design. Colonized potato dextrose agar (PDA) blocks (diameter, $7 \mathrm{~mm}$ ) were excised using a circular cutter and placed on the abaxial surface of each leaf piece. The leaves were incubated at $25^{\circ} \mathrm{C}$ for $72 \mathrm{~h}$ in a chamber with continuous 
light. The filter paper was kept moist with sterile water. After $72 \mathrm{~h}$, the length and width of the lesions within each leaf piece were measured using Image $J$ Fiji software $(\mathrm{NIH}$, Bethesda, MD, USA) and the approximate percentage of the leaf covered with lesions was calculated as previously described (Prasad and Eizenga, 2008; Eizenga et al. 2002). To analyze the $R$. solani AG1-IA infection-mediated expression of the IDD genes, onemonth-old wild-type plants were inoculated with $R$. solani AG1-IA, and their leaves were sampled after 0 , 24,48 , and $72 \mathrm{~h}$ of inoculation. The accession numbers in Genbank are as follows: IDD3 (EEC85036), IDD5 (XP_015647948), IDD10 (KAB8096499), IDD13 (XP 015610838), and LPA1 (IDD14) (XP_015629419).

\section{RNA Extraction and Quantitative Real-Time (qRT)-PCR Analysis}

Total RNA was isolated from the one-month-old rice leaves using the TRIzol reagent (Takara, Dalian, China), and the genomic DNA was removed by treatment with RQ-RNase free DNase (Promega, Madison, WI, USA). Complementary DNA was synthesized using the GoScript Reverse Transcription Kit (Promega) following the manufacturer's instructions. A BIO-RAD CFX96 Real-time PCR system (Bio-Rad, Hercules, CA, USA) and SYBR-Green (Takara) were used for the qRT-PCR analyses. The gene expression levels were normalized to that of the level of Ubiquitin. The primers used for qRTPCR are listed in Additional file 4: Table S1.

\section{Plasmid Construction}

To generate IDD3-GFP and IDD13-GFP overexpression transgenic plants, IDD3 and IDD13 ORF sequences were amplified and cloned into BglII and SpeI restriction enzyme sites of the pCAMBIA1302 binary vector, in which IDD3 or IDD13 coding sequences were N-terminally fused to the GFP coding sequences. To generate IDD13 $R N A i$ plants, $300 \mathrm{bp}$ of the IDD13 coding region was cloned into SwaI and $A s c \mathrm{I}$ sites in the sense and $X b a \mathrm{I}$ and $B a m H I$ sites in the antisense orientation, respectively, in the pFGC5941 binary vector (ChromDB).

\section{Yeast Two-Hybrid Assay}

To test the interaction between LPA1 and IDD13, IDD3 or IDD10, the Gal4 DNA-binding domain (BD) was Nterminally fused to $L P A 1$, while IDD13, IDD3, or IDD10 ORFs were cloned into the pGAD424 vector. The pair of IDDs was further transformed in the yeast strain PJ69-4A (Clontech, http://www.clontech.com/). Yeast cells carrying a pair of IDDs were grown on SD/Trp-/Leu- and SD/Trp -/Leu-His- plates. The sequences of the primers for cloning the IDD13 ORF are listed in Additional file 3: Table S1.

\section{Split GFP Assay}

The N-proximal half of YFP (nYFP) and the C-proximal half of CFP (cCFP) sequences were fused to the $\mathrm{C}$ terminal sequences of LPA1 (IDD14) and C-terminal sequences of IDD3 or IDD13 in the pXNGW and pXCGW vectors, respectively. Agrobacterium cells (GV3101) harboring half of the YFP parts were mixed and then infiltrated into Nicotiana benthamiana leaves. Before observing the YFP signal using a confocal microscope (Olympus X1000, Japan), the tobacco plants were grown in a growth chamber for 36 to $48 \mathrm{~h}$ (Kim et al. 2009a).

\section{Co-Immunoprecipitation (co-IP) and Western Blot Analyses}

IDD3-Myc + LPA1-GFP, IDD13-Myc + LPA1-GFP, IDD10-Myc + LPA1-GFP, IDD3-HA + IDD13-Myc, or IDD3-HA + IDD13-Myc + LPA1-GFP were coexpressed in $N$. benthamiana leaves, respectively. After $36 \mathrm{~h}$ of expression, the protein was extracted, and Co-IP assays were performed as described previously (Kim et al. 2009b). Twenty micrograms of protein from each sample were separated on a 10\% SDS-PAGE gel and electrotransferred onto Immobilon-P Transfer Membranes (MILLIPORE JAPAN, Tokyo, Japan). For the subsequent western blot analysis, the following primary antibodies were used: an anti-HA antibody (1:2000; Abcam, Cambridge, MA, USA), anti-GFP antibody (1:2000; Abcam), and anti-Myc antibody (1:2000; Abcam). The membranes were incubated for an additional hour with an anti-mouse or anti-rabbit horseradish peroxidase (HRP)conjugated secondary antibody (1:2000; Cell Signaling Technology, Danvers, MA, USA) before the signal was detected using an ECL Western Blotting Detection System (GE Healthcare, Piscataway, NJ, USA).

\section{Chromatin-Immunoprecipitation (ChIP) Assay}

Eight grams of rice calli were collected from transgenic plants expressing 35S:IDD13-GFP and 35S:IDD3-GFP for the ChIP assay. The ChIP assay and subsequent ChIPPCR assays were followed by a protocol described previously (Je et al. 2010). The primers used for the ChIPPCR are listed in Additional file 3: Table S1.

\section{Electrophoretic Mobility Shift Assay (EMSA)}

To produce IDD13 and IDD3 recombinant proteins, the open reading frame sequences of IDD13 and IDD3 were sub-cloned into the $p G E X 5 X-1$ expression vector, and the resulting $p G E X 5 X-1-I D D 13$ and $p G E X 5 X-1-I D D 3$ plasmids were used to transform Escherichia coli strain BL21 DE3. Recombinant proteins were harvested after a $4 \mathrm{~h}$ treatment with $0.5 \mathrm{mM}$ isopropyl $\beta$-D-1-thiogalactopyranoside (IPTG) at $28^{\circ} \mathrm{C}$. The EMSA was performed as previously described (Je et al. 2010). The primers used to obtain the EMSA probes are listed in Additional file 3: Table S1. 


\section{Transient Expression Assay}

For the transient assay, the effector plasmids (35S:LPA1, 35S:IDD13, and 35S:IDD3) and reporter (pPIN1a or mutated promoter, mpPIN1a), as well as an internal control plasmid (35S:LUC), were co-transformed into protoplast cells (Yamaguchi et al. 2010). The GUS activity analyses were performed as previously described (Xuan et al. 2013). The luciferase assay was performed using a Luciferase Assay Kit (Promega), and PEG-mediated transformation and luciferase activity assays were performed as previously described (Yoo et al. 2007). The primers used for the transient assay are listed in Additional file 3: Table S1.

\section{IAA Measurement}

The leaves from 1-month-old IDD13 OX2, IDD13 OX5, and wild-type plants were used for IAA extraction. IAA extraction and calculation methods were followed as described by Pan et al. (2010). IAA-[ $\alpha, \alpha-D 2]$ was used as an internal standard of IAA in the experiments.

\section{Statistical Analyses}

Statistical analyses were performed using Prism 5.0 (GraphPad, San Diego, CA, USA). For multiple lines comparison, a one-way analysis of variance (ANOVA) was performed, followed by Bonferroni's multiple comparison tests. Differences among the samples were considered significant at $P<0.05$.

\section{Supplementary information}

Supplementary information accompanies this paper at https://doi.org/10 1186/s12284-020-0371-1.

Additional file 1: Figure S1. PIN1a expression in IDD3 and IDDI3 mutants and overexpressors. (A) Relative expression of PIN1a in wild-type (WT), idd3-1, idd3-2, IDD3 OX \#2, and IDD3 OX \#4 plant leaves. (B) Relative expression of PIN1a in wild-type (WT), IDD13 RNAi (\#1 and \#4), IDD3 OX \#2, and IDD3 OX \#5 plant leaves. The mRNA levels of the samples were normalized to that of Ubiquitin mRNA. Data represent the means \pm standard error $(n=3)$. The expression of PIN1a in the WT was defined as "1". Different letters indicate significant differences at $P<0.05$.

Additional file 2: Figure S2. PIN1a expression in LPA1 and IDD13 genetic combinations. (A) Relative expression of PIN1a in the wild-type (WT), IDD13 RNAi, Ipa1, IDD13 RNAi/lpa1and LPA1 repressor plant leaves after $72 \mathrm{~h}$ of Rhizoctonia solani inoculation. (B) Relative expression of PIN1a in the wild-type (WT), Ipa1, IDD3 OX, and Ipa1/IDD3 OX plant leaves after $72 \mathrm{~h}$ of $R$. solani inoculation. The mRNA levels of the samples were normalized to that of Ubiquitin mRNA. Data represent the means \pm standard error $(n=3)$. The expression of PINla in WT was defined as "1". Different letters indicate significant differences at $P<0.05$.

Additional file 3: Figure S3. Measurement of the IAA content in WT and IDD13 overexpressors. The contents of IAA from the leaves of 1month-old WT and IDD13 OX lines (OX2 and OX5) were measured. Vertical bars indicate average values \pm SE $(n=3)$. Different letters indicate significant differences at $P<0.05$.

Additional file 4: Table S1. Primer sequences

\section{Abbreviations}

ChIP: Chromatin-Immunoprecipitation; DIC: Differential Interference Contrast; EMSA: Electrophoretic Mobility Shift Assay; IDD: Indeterminate Domain;
LPA1: Loose Plant Architecture1; OX: Overexpressor; PIN1a: Pin-Formed 1a; ShB: Sheath Blight Disease; WT: Wild Type

\section{Acknowledgements}

The authors would thank Dr. Chang-deok Han at Gyeongsang National University, Korea for providing rice seeds.

\section{Authors' Contributions}

Q Sun, and YH Xuan designed the experiment and wrote the manuscript. Q Sun, DD Li, J Chu, DP Yuan, and X Han conducted the experiments and performed data analysis. S Li, and $\sqcup$ Zhong participated in material development, sample preparation and data analysis. Q Sun, X Han, and YH Xuan corrected the manuscript. All authors read and approved the final manuscript.

\section{Funding}

This work was supported by an initiative grant (880416008) from Shenyang Agricultural University, the Support Plan for Innovative Talents in Colleges and Universities of Liaoning Province (LR2017037), and National Key R\&D Program of China (2016YFD0101004).

\section{Availability of Data and Materials}

The datasets supporting the conclusions of this article are provided within the article and its additional files.

Ethics Approval and Consent to Participate Not applicable.

\section{Consent for Publication}

Not applicable.

\section{Competing Interests}

The authors declare that they have no competing interests.

\section{Author details}

${ }^{1}$ College of Plant Protection, Shenyang Agricultural University, Shenyang 110866, China. ${ }^{2}$ Institute of Plant Protection, Liaoning Academy of Agricultural Sciences, Shenyang 110161, China. ${ }^{3}$ Shaanxi Key Laboratory of Chinese Jujube, Yan'an University, Yan'an 716000, Shaanxi, China. ${ }^{4}$ College of Life Science, Yan'an University, Yan'an 716000, Shaanxi, China. ${ }^{5}$ Microbial Research Institute, Liaoning Academy of Agricultural Sciences, Chaoyang 122000, China. ${ }^{6}$ College of Biological Science and Engineering, Fuzhou University, Fuzhou 350108, China.

Received: 24 October 2019 Accepted: 28 January 2020

Published online: 06 March 2020

\section{References}

Adamowski M, Friml J (2015) PIN-dependent auxin transport: action, regulation, and evolution. Plant Cell 27(1):20-32

Chen Z, Agnew JL, Cohen JD, He P, Shan L, Sheen J, Kunkel BN (2007) Pseudomonas syringae type III effector AvrRpt2 alters Arabidopsis thaliana auxin physiology. Proc Natl Acad Sci U S A 104(50):20131-20136

Colasanti J, Yuan Z, Sundaresan V (1998) The indeterminate gene encodes a zinc finger protein and regulates a leaf-generated signal required for the transition to flowering in maize. Cell 93(4):593-603

Cui D, Zhao J, Jing Y, Fan M, Liu J, Wang Z, Xin,W, Hu Y (2013) The arabidopsis IDD14, IDD15, and IDD16 cooperatively regulate lateral organ morphogenesis and gravitropism by promoting auxin biosynthesis and transport. PLoS Genet 9(9):e1003759

Dou M, Cheng S, Zhao B, Xuan Y, Shao M (2016) The indeterminate domain protein $\mathrm{ROC} 1$ regulates chilling tolerance via activation of DREB1B/CBF1 in Rice. Int J Mol Sci 17(3):233

Eizenga GC, Lee FN, Rutger JN (2002) Screening Oryza species plants for Rice sheath blight resistance. Plant Dis 86(7):808-812

Feurtado JA, Huang D, Wicki-Stordeur L, Hemstock LE, Potentier MS, Tsang EW, Cutler AJ (2011) The Arabidopsis C2H2 zinc finger INDETERMINATE DOMAIN1/ ENHYDROUS promotes the transition to germination by regulating light and hormonal signaling during seed maturation. Plant Cell 23(5):1772-1794

Fu J, Liu H, Li Y, Yu H, Li X, Xiao J, Wang S (2011) Manipulating broad-spectrum disease resistance by suppressing pathogen-induced auxin accumulation in rice. Plant Physiol 155(1):589-602 
Gao Y, Zhang C, Han X, Wang ZY, Ma L, Yuan P, Wu JN, Zhu XF, Liu JM, Li DP, Hu YB, Xuan YH (2018) Inhibition of OsSWEET11 function in mesophyll cells improves resistance of rice to sheath blight disease. Mol Plant Pathol 19(9):2149-2161

Helliwell EE, Wang Q, Yang Y (2013) Transgenic rice with inducible ethylene production exhibits broad-spectrum disease resistance to the fungal pathogens Magnaporthe oryzae and Rhizoctonia solani. Plant Biotechnol J 11(1):33-42

Huang P, Yoshida H, Yano K, Kinoshita S, Kawai K, Koketsu E, Hattori M, Ordonio RL, Matsuoka M, Ueguchi-Tanaka M (2018) OsIDD2, a zinc finger and INDETERMINATE DOMAIN protein, regulates secondary cell wall formation. J Integr Plant Biol 60(2):130-143

Je BI, Piao HL, Park SJ, Park SH, Kim CM, Xuan YH, Park SH, Huang J, Do Choi Y, An G, Wong HL, Fujioka S, Kim MC, Shimamoto K, Han CD (2010) RAV-Like1 maintains brassinosteroid homeostasis via the coordinated activation of BRI1 and biosynthetic genes in rice. Plant Cell 22(6):1777-1791

Kim JG, Li X, Roden JA, Taylor KW, Aakre CD, Su B, Lalonde S, Kirik A, Chen Y, Baranage G, McLane H, Martin GB, Mudgett MB (2009a) Xanthomonas T3S effector XopN suppresses PAMP-triggered immunity and interacts with a tomato atypical receptor-like kinase and TFT1. Plant Cell 21(4):1305-1323

Kim TW, Guan S, Sun Y, Deng Z, Tang W, Shang JX, Sun Y, Burlingame AL, Wang ZY (2009b) Brassinosteroid signal transduction from cell-surface receptor kinases to nuclear transcription factors. Nat Cell Biol 11(10):1254-1260

Kouzai Y, Kimura M, Watanabe M, Kusunoki K, Osaka D, Suzuki T, Matsui H, Yamamoto M, Ichinose $Y$, Toyoda K, Matsuura T, Mori IC, Hirayama T, Minami E, Nishizawa Y, Inoue K, Onda Y, Mochida K, Noutoshi Y (2018) Salicylic aciddependent immunity contributes to resistance against Rhizoctonia solani, a necrotrophic fungal agent of sheath blight, in rice and Brachypodium distachyon. New Phytol 217(2):771-783

Kozaki A, Hake S, Colasanti J (2004) The maize ID1 flowering time regulator is a zinc finger protein with novel DNA binding properties. Nucleic Acids Res 32(5):1710-1720

Liu JM, Park SJ, Huang J, Lee EJ, Xuan YH, Je BI, Kumar V, Priatama RA, Raj KV, Kim SH, Min MK, Cho JH, Kim TH, Chandran AK, Jung KH, Takatsuto S, Fujioka S, Han CD (2016) Loose plant Architecture1 (LPA1) determines lamina joint bending by suppressing auxin signalling that interacts with $C^{-22}$ hydroxylated and 6-deoxo brassinosteroids in rice. J Exp Bot 67(6):1883-1895

Maeda S, Dubouzet JG, Kondou Y, Jikumaru Y, Seo S, Oda K, Matsui M, Hirochika H, Mori M (2019) The rice CYP78A gene BSR2 confers resistance to Rhizoctonia solani and affects seed size and growth in Arabidopsis and rice. Sci Rep 9(1):587

Mao B, Liu X, Hu D, Li D (2014) Co-expression of RCH10 and AGLU1 confers rice resistance to fungal sheath blight Rhizoctonia solani and blast Magnorpathe oryzae and reveals impact on seed germination. World J Microbiol Biotechnol 30(4):1229-1238

Naseem M, Philippi N, Hussain A, Wangorsch G, Ahmed N, Dandekar T (2012) Integrated systems view on networking by hormones in Arabidopsis immunity reveals multiple crosstalk for cytokinin. Plant Cell 24(5):1793-1814

Pan X, Welti R, Wang X (2010) Quantitative analysis of major plant hormones in crude plant extracts by high-performance liquid chromatography-mass spectrometry. Nat Protoc 5:986-992

Park SJ, Kim SL, Lee S, Je BI, Piao HL, Park SH, Kim CM, Ryu CH, Park SH, Xuan YH, Colasanti J, An G, Han CD (2008) Rice indeterminate 1 (Osld1) is necessary for the expression of Ehd1 (early heading date 1) regardless of photoperiod. Plant J 56(6):1018-1029

Prasad B, Eizenga GC (2008) Rice sheath blight disease resistance identified in Oryza spp. Accessions Plant Dis 92(11):1503-1509

Robert-Seilaniantz A, Grant M, Jones JD (2011) Hormone crosstalk in plant disease and defense: more than just jasmonate-salicylate antagonism. Annu Rev Phytopathol 49:317-343

Savary S, Castilla NP, Elazegui FA, McLaren CG, Ynalvez MA, Teng PS (1995) Direct and indirect effects of nitrogen supply and disease source structure on rice sheath blight spread. Phytopathology 85(9):959-965

Savary S, Willocquet L, Elazegui FA, Castilla NP, Teng PS (2000) Rice pest constraints in tropical asia: quantification of yield losses due to rice pests in a range of production situations. Plant Dis 84(3):357-369

Seo PJ, Ryu J, Kang SK, Park CM (2011) Modulation of sugar metabolism by an INDETERMINATE DOMAIN transcription factor contributes to photoperiodic flowering in Arabidopsis. Plant J 65(3):418-429

Shah JM, Raghupathy V, Veluthambi K (2009) Enhanced sheath blight resistance in transgenic rice expressing an endochitinase gene from Trichoderma virens. Biotechnol Lett 31(2):239-244

Sun Q, Li TY, Li DD, Wang ZY, Li S, Li DP, Han X, Liu JM, Xuan YH (2019) Overexpression of loose plant architecture 1 increases planting density and resistance to sheath blight disease via activation of PIN-FORMED 1a in rice. Plant Biotechnol J 17(5):855-857

Volz R, Kim SK, Mi J, Mariappan KG, Siodmak A, Al-Babili S, Hirt H (2019) A chimeric IDD4 repressor constitutively induces immunity in Arabidopsis via the modulation of salicylic- and jasmonic acid homeostasis. Plant Cell Physiol 60(7):1536-1555

Wang R, Lu L, Pan X, Hu Z, Ling F, Yan Y, Liu Y, Lin Y (2015) Functional analysis of OsPGIP1 in rice sheath blight resistance. Plant Mol Biol 87(1-2):81-191

Welch D, Hassan H, Blilou I, Immink R, Heidstra R, Scheres B (2007) Arabidopsis JACKDAW and MAGPIE zinc finger proteins delimit asymmetric cell division and stabilize tissue boundaries by restricting SHORT-ROOT action. Genes Dev 21(17):2196-2204

Wu X, Tang D, Li M, Wang K, Cheng Z (2013) Loose plant Architecture1, an INDETERMINATE DOMAIN protein involved in shoot gravitropism, regulates plant architecture in rice. Plant Physiol 161(1):317-329

Xuan YH, Priatama RA, Huang J, Je BI, Liu JM, Park SJ, Piao HL, Son DY, Lee JJ, Park SH, Jung KH, Kim TH, Han CD (2013) Indeterminate domain 10 regulates ammonium-mediated gene expression in rice roots. New Phytol 197(3):791-804

Yamaguchi M, Ohtani M, Mitsuda N, Kubo M, Ohme-Takagi M, Fukuda H, Demura T (2010) VND-INTERACTING2, a NAC domain transcription factor, negatively regulates xylem vessel formation in Arabidopsis. Plant Cell 22(4):1249-1263

Yoo SD, Cho YH, Sheen J (2007) Arabidopsis mesophyll protoplasts: a versatile cell system for transient gene expression analysis. Nat Protoc 2(7):1565-1572

Yuan P, Zhang C, Wang ZY, Zhu XF, Xuan YH (2018) RAVL1 activates brassinosteroids and ethylene signaling to modulate response to sheath blight disease in rice. Phytopathol 108(9):1104-1113

Zazimalova E, Murphy AS, Yang H, Hoyerova K, Hosek P (2010) Auxin transporters--why so many? Cold Spring Harb Perspect Biol 2(3):a001552

\section{Publisher's Note}

Springer Nature remains neutral with regard to jurisdictional claims in published maps and institutional affiliations.

\section{Submit your manuscript to a SpringerOpen ${ }^{\circ}$ journal and benefit from:}

- Convenient online submission

- Rigorous peer review

- Open access: articles freely available online

High visibility within the field

- Retaining the copyright to your article

Submit your next manuscript at $>$ springeropen.com 